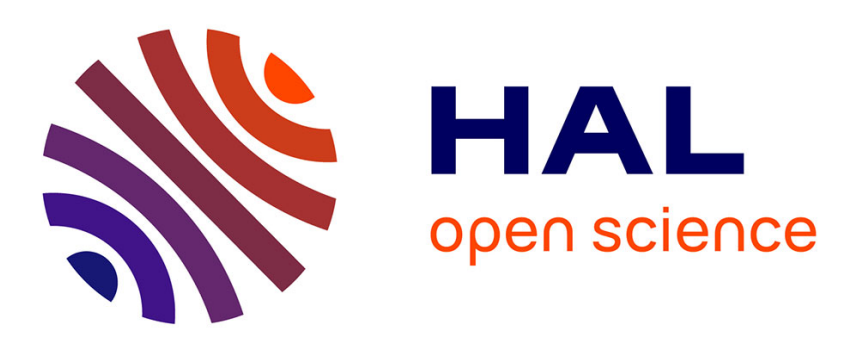

\title{
A multi-stream Vlasov modeling unifying relativistic Weibel-type instabilities
}

A. Inglebert, A. Ghizzo, T. Reveille, D. del Sarto, Pierre Bertrand, F. Califano, D Del Sarto

\section{- To cite this version:}

A. Inglebert, A. Ghizzo, T. Reveille, D. del Sarto, Pierre Bertrand, et al.. A multi-stream Vlasov modeling unifying relativistic Weibel-type instabilities. EPL - Europhysics Letters, 2011, 95 (4), pp.45002. 10.1209/0295-5075/95/45002 . hal-01791693

\section{HAL Id: hal-01791693 \\ https://hal.univ-lorraine.fr/hal-01791693}

Submitted on 20 May 2018

HAL is a multi-disciplinary open access archive for the deposit and dissemination of scientific research documents, whether they are published or not. The documents may come from teaching and research institutions in France or abroad, or from public or private research centers.
L'archive ouverte pluridisciplinaire HAL, est destinée au dépôt et à la diffusion de documents scientifiques de niveau recherche, publiés ou non, émanant des établissements d'enseignement et de recherche français ou étrangers, des laboratoires publics ou privés. 


\title{
A multi-stream Vlasov modeling unifying relativistic Weibel-type instabilities
}

\author{
A. Inglebert ${ }^{1,2}$, A. Ghizzo $^{1}$, T. Reveille ${ }^{1}$, D. Del Sarto ${ }^{1}$, \\ P. Bertrand ${ }^{1}$, F. Califano ${ }^{2}$ \\ Address: 1. IJL UMR 7198, University of Lorraine, \\ BP 239 F-54506 Vandoeuvre les Nancy, France. and \\ 2: Physics Department, University of Pisa, Pisa, Italy
}

(Dated:)

We present a multi-stream model obtained from the Vlasov-Maxwell system of equations based on the invariance of the canonical momentum in the perpendicular direction in a $1 \mathrm{D}-2 \mathrm{~V}$ phase space. The model is conceived for the study of the Weibel and current filamentation instability in the relativistic regime, but turns out to be of more general importance. It can be used numerically for the study of the non linear, kinetic dynamics with a drastic reduction of the computational cost with respect to the integration of the corresponding Vlasov-Maxwell system.

The origin of magnetic fields observed in laboratory and astrophysical plasmas is a challenging problem in plasma physics. In this context, the Weibel instability [1] is of key importance, as witnessed by the impressive number of papers appeared in these last few years. This instability, starting from an initial temperature anisotropy, generates "quasi-static" magnetic fields through the redistribution of currents in space that grow more and more separated by the self-consistent electromagnetic fields [2]. The typical scale length of the generated magnetic fields is the electron skin depth.

Magnetic fields can also be generated by a similar process, the Current Filamentation instability (CFI), driven by electron momentum anisotropy of counter-streaming electron populations. Any infinitesimal transverse perturbation separates these currents that repeal each other, thus reinforcing the initial perturbation. As a result, the system generates magnetic field in a way similar to the Weibel case [3-5]. These instabilities share the common point of phase space anisotropy and present mathematical analogies and similar behaviors, as for instance the possibility of generating magnetic fields by extracting the free energy from a velocity distribution function anisotropy [6-8].

This aspect underlies the similarity between Weibel and CFI instabilities and allows us to built a new theoretical model starting from the possibility of representing temperature anisotropy by means of a finite number of counter streaming beams. This "multi-streams" model turns out to be of more general importance and not limited to the Weibel case only.

We underline that, as a consequence of the strong physical and mathematical analogies, the CFI instability has been often named Weibel instability, in particular in the laser-plasma interaction context. Furthermore, the Weibel instability can be described, in the multi-stream model, by at least three streams in the perpendicular momentum space. More generally, it is always possible to describe any perpendicular temperature effect by several streams. Therefore, for the sake of simplicity, in 
the following we shall refer to a "Weibel-type instability" as to any instability driven by phase space anisotropy generating magnetic field.

In laser plasmas, the CFI typically occurs as a consequence of the acceleration of a "hot" electron bunch that induces a colder, although denser return current in order to maintain quasi-neutrality [9]. The corresponding self-consistently generated magnetic fields, play a key role in the strongly non linear relativistic dynamics [10, 11], in the scattering at the critical surface contributing to beam divergence [12] and in the expansion of plasma into vacuum [13].

The Weibel and/or CFI is also often invoked in Astrophysics where it has been proposed as the source of a seed magnetic field [14] at a cosmological level [15]. In addition, it plays a basic role in the dynamics of relativistic collisionless shocks [16] associated with gamma ray bursts [17].

From a theoretical point of view, the relativistic theory [18], recently revised for a beam-plasma system in the linear, kinetic limit [19], is still not completely satisfying as in the non-relativistic limit [20].

For these reasons, it is important to improve the models to be numerically exploited in order to address the non linear, collisionless relativistic regime, starting from any possible initial condition representing an anisotropic distribution state in phase space. In this perspective, the present work considers an unified Vlasov model, allowing to treat electromagnetic Weibel-type instabilities where kinetic effects play a key role. Models using a reduced phase space dimension are of interest for the understanding of the physical processes underlying the plasma dynamics of "real" systems.

Here we restrict our analysis to plane waves propagating along the $x$-direction assuming ions as a fixed neutralizing background. Let us consider the Hamiltonian of one particle, of rest mass $m$ and charge $e$, in the electromagnetic field $(\boldsymbol{E}, \boldsymbol{B})$, which in the relativistic regime reads $H=m c^{2}(\gamma-1)+e \phi(x, t)$. By using the canonical momentum $\boldsymbol{P}_{c}=\boldsymbol{p}+e \boldsymbol{A}_{\perp}$, the Lorentz factor can be expressed as $\gamma=\left[1+\left(\boldsymbol{P}_{c}-e \boldsymbol{A}_{\perp}(x, t)\right)^{2} / m^{2} c^{2}\right]^{1 / 2}$, where $\phi$ and $\boldsymbol{A}$ denote the electrostatic potential and the vector potential, respectively. In the Coulomb gauge $(\operatorname{div} \boldsymbol{A}=0), \boldsymbol{A}_{\perp} \boldsymbol{A}_{\perp}(x, t)$ and the Hamilton equation is $d \boldsymbol{P}_{c} / d t=-\partial H / \partial \boldsymbol{q}$. Since we are limited to plane waves propagating in the $x-$ direction, the Hamilton equation splits in the longitudinal and perpendicular directions, $d P_{c x} / d t=-\partial H / \partial x$ and $d \boldsymbol{P}_{c \perp} / d t=$ $-\partial H / \partial \boldsymbol{q}_{\perp}=0$. Thus, owing to the invariance of the perpendicular generalized canonical momentum, it is possible to represent the plasma evolution by a class of initial conditions invariant under the dynamics. This approach is analogous to the one used for a drift-kinetic model in Ref. [21], which has been exploited also in the framework of magnetized plasmas to investigate the process of magnetic reconnection [22].

Without loss of generality, we can consider a plasma, where the particles are divided into $N$ bunches of particles, each "stream" $j$ (with $j=1, \ldots, N$ ) having the same initial perpendicular momentum $\boldsymbol{P}_{c \perp}=\boldsymbol{C}_{j}=$ const. We can now define, for a particle population $j$, a reduced Vlasov-type equation and a distribution function $f_{j}\left(x, p_{x}, t\right)$. The corresponding Hamiltonian of one particle of stream $j$ is then given by $H_{j}=m c^{2}\left(\gamma_{j}-1\right)+e \phi(x, t)$, where the Lorentz factor can be expressed as $\gamma_{j}=\left[1+p_{x}^{2} / m^{2} c^{2}+\left(\boldsymbol{C}_{j}-e \boldsymbol{A}_{\perp}(x, t)\right)^{2} / m^{2} c^{2}\right]^{1 / 2}$.

The distribution function $f_{j}$ satisfies the reduced Vlasov equation, for $j=1, \ldots, N$ : 


$$
\frac{\partial f_{j}}{\partial t}+\frac{p_{x}}{m \gamma_{j}} \frac{\partial f_{j}}{\partial x}+\left(e E_{x}-\frac{1}{2 m \gamma_{j}} \frac{\partial}{\partial x}\left(\boldsymbol{C}_{j}-e \boldsymbol{A}_{\perp}\right)^{2}\right) \frac{\partial f_{j}}{\partial p_{x}}=0
$$

For each population $j$, we define the stream density as $n_{j}(x, t)=\int f_{j} d p_{x}$ and the current density as $\boldsymbol{J}_{\perp j}=$ $e\left(\boldsymbol{C}_{j}-e \boldsymbol{A}_{\perp}\right) \rho_{j} / m$ with a relativistic density $\rho_{j}=\int f_{j} / \gamma_{j} d p_{x}$. Our kinetic multi-stream model is given by $N$ reduced Vlasov equations of type (1), for each stream $j$, self-consistently coupled to the longitudinal electric field $E_{x}$ and the potential vector $\boldsymbol{A}_{\perp}(x, t)$, allowing to reduce the $4 \mathrm{D}$ phase space into a $2 \mathrm{D}$ one (plus $2 N$ values for the corresponding $\left.\boldsymbol{C}_{j}\right)$. The Maxwell-Gauss equation is rewritten in the form $\partial E_{x} / \partial x=\left(e / \varepsilon_{0}\right)\left(n(x, t)-n_{0}\right)$ and the potential vector satisfies the equation $\partial^{2} \boldsymbol{A}_{\perp} / \partial t^{2}-c^{2} \partial^{2} \boldsymbol{A}_{\perp} / \partial x^{2}=\left(1 / \varepsilon_{0}\right) \boldsymbol{J}_{\perp}$ Here the coupling with the multi-stream model is made through the source terms $n=\sum_{j} n_{j}$ and $\boldsymbol{J}_{\perp}=\sum_{j} \boldsymbol{J}_{\perp j}$.

We now focus on the possibility of deriving a generalized dispersion relation of Weibel-type in the fluid approximation. By assuming cold streams, $f_{j}\left(x, p_{x}, t\right)=n_{j}(x, t) \delta\left(p_{x}-u_{j}(x, t)\right)$, and considering the moments of the Vlasov equation (1), it is possible to build up a closed multi-fluid model in the form:

$$
\begin{gathered}
\frac{\partial u_{j}}{\partial t}+\frac{u_{j}}{m \widetilde{\gamma_{j}}} \frac{\partial u_{j}}{\partial x}=e E_{x}-\frac{1}{2 m \widetilde{\gamma}_{j}} \frac{\partial}{\partial x}\left(C_{j}-e A_{y}\right)^{2} \\
\frac{\partial n_{j}}{\partial t}+\frac{\partial}{\partial x}\left(\frac{n_{j} u_{j}}{m \widetilde{\gamma_{j}}}\right)=0
\end{gathered}
$$

We consider a linearly polarized electromagnetic plane wave (with $\boldsymbol{P}_{c \perp}=C_{j} \boldsymbol{e}_{y}=$ const and $\boldsymbol{A}_{\perp}=A_{y}(x, t) \boldsymbol{e}_{y}$ ). The term $\widetilde{\gamma}_{j}$ represents the mean Lorentz factor obtained from the standard expression by replacing the variable $p_{x}$ by the quantity $u_{j}$ representing the longitudinal momentum of the stream $j$ in the fluid approximation. We consider an expansion around an equilibrium characterized by a mean density $n_{0 j}$ and a mean longitudinal momentum $u_{0 j}=0$ and assume $\sum_{j=1, N} n_{0 j}=n_{0}$ and $\sum_{j=1, N} C_{j} n_{0 j} / \Gamma_{0 j}=0$, corresponding at zero order to the neutrality condition and to the requirement that the initial net current is zero, respectively. Here $\Gamma_{0 j}$ takes into account, at zeroorder, the pure transverse contribution in the expression of the Lorentz factor $\Gamma_{0 j}=\left[1+C_{j}^{2} / m^{2} c^{2}\right]^{1 / 2}$. By linearizing and performing a time / space Fourier transform, we get the dispersion relation for Weibel-type instabilities:

$$
\begin{gathered}
\left(1-\sum_{j=1}^{N} \frac{\omega_{p j}^{2}}{\omega^{2} \Gamma_{0 j}}\right)\left\{-\omega^{2}+k^{2} c^{2}+\sum_{j=1}^{N} \frac{\omega_{p j}^{2}}{\Gamma_{0 j}^{3}}\right. \\
\left.+\frac{k^{2} c^{2}}{\omega^{2}} \sum_{j=1}^{N} \frac{\omega_{p j}^{2}}{\Gamma_{0 j}^{3}} \frac{C_{j}^{2}}{m^{2} c^{2}}\right\}=-\frac{k^{2} c^{2}}{\omega^{4}}\left(\sum_{j=1}^{N} \frac{\omega_{p j}^{2}}{\Gamma_{0 j}^{2}} \frac{C_{j}}{m c}\right)^{2}
\end{gathered}
$$


In Eq. (4) the term $\omega_{p j}^{2}=n_{0 j} e^{2} / m \varepsilon_{0}$ is the "plasma frequency" of the particle bunch $j$. The analytical formulation of the linear dispersion relation, Eq. (4), as a discrete summation over an assembly of streams provides a general and exact approach able to take into account any anisotropy of the distribution function, even in the relativistic regime. The model represents an exact reduction of the phase space dimension (elimination of the transverse momentum variable $\boldsymbol{p}_{\perp}$ ) since the multi-stream approach makes use of the transverse canonical momentum invariance. The transverse momentum is hidden in the various streams $j$ (with $j=1, \ldots, N$ ). For a precise description of a continuous distribution, larger values of $N$ would be needed. Nevertheless, interesting results can still be obtained with $N$ as small as 1,2 or 3 . The case $N=1$ corresponds to a cold (Dirac-type) distribution function in the perpendicular direction and this choice has been intensively used in Refs. [23, 24] to describe laser-plasma instabilities as parametric Raman-type instability in the relativistic regime. The more interesting cases $N=2$ and/or $N=3$ can be used to describe Weibel-type instabilities. In particular, the dispersion relation, Eq. (4), with $N=2$ reduces to the particular case of the CFI case in the relativistic regime in agreement with previous results in Ref. [25].

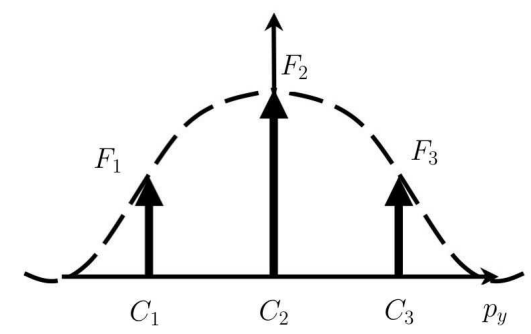

Figure 1: $p_{y}$ for the two counter-streaming filamentation instability.

A relevant feature is the fact that Eq. (4) contains only linear and quadratic terms in $C_{j}$. This suggests that our model is well suited to describe physical systems that can be modeled by a limited number of moments as density, pressure and "temperature". Indeed we can consider to define the term $\sum_{j=1}^{N} \omega_{p j}^{2} C_{j}^{2} / \Gamma_{0 j}^{3} m^{2} c^{2}$ as equivalent to a "relativistic temperature".

For instance in the non relativistic limit we recover the well-known case of the Weibel instability [1] driven by a temperature anisotropy by taking $N=3$ streams (see Fig. 1). For this purpose, it is necessary to initialize the system in the form of a distribution function given by $F\left(x, p_{x}, p_{y}\right)=n_{0} \delta\left(p_{x}\right) \sum_{j=1,3} F_{j} \delta\left(p_{y}-C_{j}\right)$, where $F_{j}$ is a normalization constant. We assume a symmetric distribution: $C_{2}=0, F_{1}=F_{3}$ and $C_{1}=C_{3}$. The non relativistic limit is recovered by taking the limit $\Gamma_{0 j} \rightarrow 1$ in Eq. (4), where now the second term tends to zero (corresponding to the zero net current condition $\left.\sum_{j=1,3} C_{j} F_{j}=0\right)$.

As a result, we obtain the dispersion relation of a Weibel-type instability in the usual form $k^{2} c^{2}+\omega_{p}^{2}-\omega^{2}+$ $\left(k^{2} c^{2} / \omega^{2}\right) \sum_{j=1}^{3} \omega_{p j}^{2} C_{j}^{2} / m^{2} c^{2}=0$. In particular, starting from a Maxwellian distribution, we get $F_{1}=F_{3}=1 / 6$ and $F_{2}=2 / 3$, and $C_{1}=C_{3}=3^{1 / 2} m v_{t h}$, where $v_{t h}$ is the thermal velocity. Finally, still in the non relativistic limit, the $C_{j}$ quadratic sum $k^{2} c^{2} / \omega^{2} \sum_{j=1,3} \omega_{p j}^{2} C_{j}^{2} / m^{2} c^{2}$ reduces to $k^{2} v_{t h}^{2} / \omega^{2}$. We point out that, from a numerical point of view, 
our reduced model allows for a drastic reduction of the computational time when only a small number of streams is required. It is well-known that a full 3D kinetic approach, based on non-particle (Vlasov) codes, is today at the limits of computer capabilities. Thus reduced models are also of interest in high performance computing. By introducing only three streams in our model, the multi-stream model allows us to introduce a perpendicular temperature and so to represent any temperature anisotropy effect.

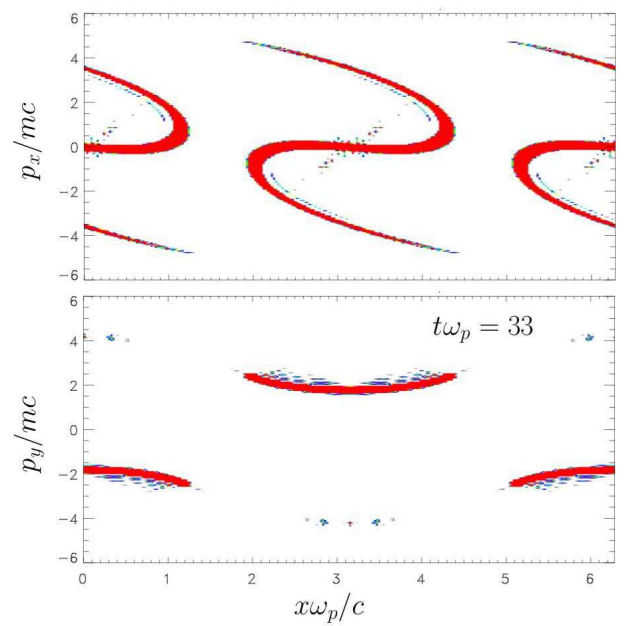

Figure 2: $\left(x, p_{x}\right)$ and $\left(x, p_{y}\right)$ phase space averaged along $p_{y}$ (top panel) and $p_{x}$ (bottom panel), respectively, obtained from the $\mathrm{V}$-model at $t=33$ in the case of the current filamentation instability.

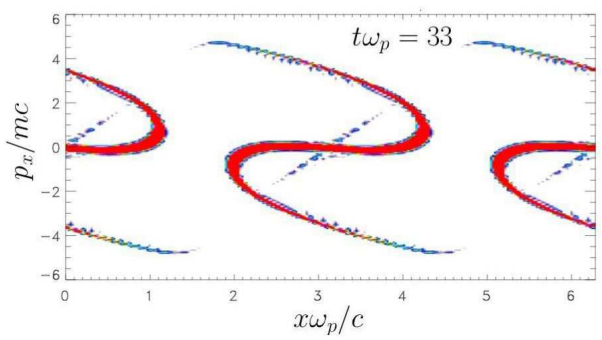

Figure 3: $\left(x, p_{x}\right)$ phase space obtained from the R-model at $t=33$ in the case of the current filamentation instability. The parameters are the same as in Fig. 2.

The multi-stream model (hereafter the R-model) offers an exact description of the plasma dynamics even with a small number of streams. On the other hand, in the full Vlasov approach (hereafter the V-model), the exchange of momentum is described by a differential operator, $\partial / \partial p_{y}$, to be numerically approximated by some scheme. Therefore, a mesh in the transverse momentum space $p_{y}$ is required instead of a "limited number of streams" used in the R-model, corresponding to a saving factor of order $N_{p_{y}} / N$ in computer time with respect to the V-model. We focus now on

\section{the non linear behavior of the CFI.}

In order to show the efficiency of the reduced model, numerical simulations have been performed using a semiLagrangian Vlasov solver, which has been presented in Ref. [26]. The Vlasov code solves the full Vlasov equation in the $1 \mathrm{D} 2 \mathrm{~V}$ phase space, denoted here as the V-model. For the multi-stream model, we solve numerically the reduced 

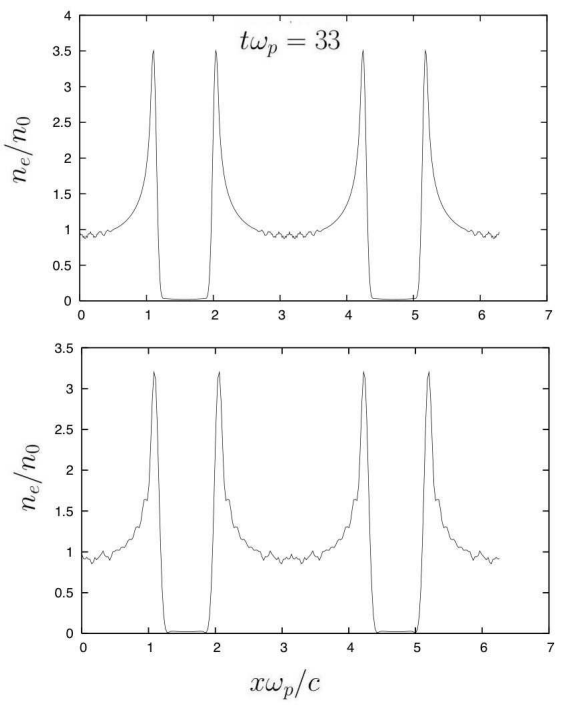

Figure 4: Top panel: Electron density behavior at time $t=33$ for CFI using the R-model. Bottom panel: the corresponding density in the physical space obtained by the global V-model.

Vlasov equation given by Eq. (1) in the 1D1V phase space. This multi-stream model is denoted here by the R-model. Both models are self-consistently coupled to the Maxwell equations. The initial distribution condition is composed by two Maxwellians centered at $\boldsymbol{p}_{01} / m c=-3 \boldsymbol{e}_{y}$ and $\boldsymbol{p}_{02} / m c=3 \boldsymbol{e}_{y}$. The beam densities are $n_{01}=n_{02}=0.5 n_{0}$ corresponding to a "symetric" case. Here we focus the attention on purely initial sine perturbation (on the magnetic $B_{z^{-}}$component) with wave vector $\boldsymbol{k}_{0}=k_{0} \boldsymbol{e}_{x}$ along the $x$ - direction, perpendicular to the two counter-streaming electron beams. At $t=0$ we perturb the system using small perturbations of vector potential amplitude $\epsilon \simeq 10^{-4}$ and we take $k_{0} c / \omega_{p}=1$. The electron temperature is chosen as $T_{e}=2 \mathrm{keV}$ in both the $p_{x}$ and $p_{y}$ direction in the $\mathrm{V}$-model while, in the R-model, only the $p_{x}$ direction has a non zero temperature and equal to that of the V-model. The phase space sampling used in the V-model is $N_{x} N_{p_{x}} N_{p_{y}}=256 \times 257^{2}$, corresponding to $1.69 \times 10^{7}$ grid points. In the R-model, we take $N_{x} N_{p_{x}}=513^{2}$ grid points. Similar results have been obtained with a phase space sampling of $257^{2}$. The time step used in both simulations is $\Delta t \omega_{p}=0.003$.

In Fig. 2, we show the electron distribution in the $x-p_{x}$ (top panel) and $x-p_{y}$ (bottom panel) phase space, respectively, for the V-model when the instability starts to saturate. The same $x-p_{x}$ phase space dynamics, described by the R-model using the sum of the two streams $\sum_{j} f_{j}$, is shown in the top panel of Fig. 3 at the same time instant of the one obtained from the V-model and shown in Fig. 2. These figures show that the dynamics is correctly described by the R-model where only two streams are considered. An interesting result of our investigations is an electron cavitation process occurring in the region of high intensity, quasi-static magnetic fields, as shown in Fig. 4. The top panel in Fig. 4 is obtained from the multi-stream R-model, while the bottom panel shows the same diagnostic but now obtained from the V-model. In spite of very small differences in the peaks of density, the electron cavitation presents the same features in both cases.

We have check, at least for the duration of the simulations presented here, that mobile ions do not modify signifi- 
cantly the results.

In summary, we present a new model based on a multi-stream approach, using a Hamiltonian reduction technique relying on the invariance of the transverse canonical momentum. From a computational point of view, the R-model provides a saving factor of order of $N_{p_{y}} / N \sim 100$ for the considered case. Such computational gain could be much larger up to $N_{p_{\perp}} / N \sim 10^{4}$ in the case of a circularly polarized electromagnetic wave.

The model has been first applied to the study of Weibel-type instabilities in relativistic conditions without imposing significant constraints on the shape of the distribution function (which could be far from a Maxwellian one, in particular in the relativistic regime). Once again, we underline that the $\mathrm{R}$-model is capable of capturing the main features of the physics of the problem, even during the non linear regime of the instability, despite the small number of streams used. Of course, the loss of exact invariance of the perpendicular canonical momentum in two-dimensional spatial systems, precludes the generalization of the method to systems of higher spatial dimension. However, it is possible to replace the exact invariant by an adiabatic invariant when the longitudinal electrostatic instability presents a slow growth with respect to the electromagnetic Weibel instability. The extension to larger phase space dimensions will be the object of future work.

\section{Acknowledgments}

This work was granted to access to the HPC resources of [CCRT/CINES/IDRIS] under the allocation 2010 - [number of dossier] made by GENCI (Grand Equipement National de Calcul Intensif). The authors are grateful to the IDRIS computational center (Orsay, France) for computer time allocation.

[1] E. W. Weibel, Phys. Rev. Lett 2, 83 (1959).

[2] R. L. Morse, C.W. Nielson, Phys. Fluids 14, 830, (1971).

[3] B.D. Fried, Phys. Fluids 2, 337 (1959).

[4] A.R. Bell, J.R. Davies, S. Guerin, H. Ruhl, Plasma Phys. Control. Fus. 39, 653 (1997)

[5] F. Califano, F. Pegoraro, S.V. Bulanov, Phys. Rev. E 56, 963 (1997)

[6] G. Kalman, C. Montes, and D. Quemada, Phys. Fluids 11, 1797 (1968)

[7] R.C. Davidson, D.A. Hammer, I. Haber, C.E. Wagner, Phys. Fluids 15, 317 (1972)

[8] F. Califano, R. Prandi, F. Pegoraro, S.V. Bulanov, Phys. Rev. E 58, 7837 (1998)

[9] T. Liseikina, F. Califano, V. Vshivkov, F. Pegoraro, S. Bulanov, Phys. Rev. E 60, 5991 (1999)

[10] O. Polomarov, I. Kaganovich, G. Shvets, Phys. Rev. Lett. 101, 175001 (2008)

[11] F. Califano, D. Del Sarto, and F. Pegoraro, Phys. Rev. Lett. 96, 105008 (2006)

[12] A. Debayle, J.J. Honrubia, E. dHumieres, V. T. Tikhonchuk, Phys. Rev. E 82, 036405 (2010)

[13] C. Thaury, P. Mora, A. Heron, J.C. Adam, T.M. Antonsen, Phys. Rev. E 82, 026408 (2010)

[14] F. Califano, T. Cecchi, C. Chiuderi, Phys. Plasmas 9, 451 (2002) 
[15] M. Lazar, R. Schlickeiser, R. Wielebinski, S. Poedts, Ap.J. 693, 1133 (2009)

[16] K.I Nishikawa et al., Ap.J. Lett. 698, L10 (2009)

[17] M.V. Medvedev, O.V. Zakutnyaya, Ap.J. Lett. 696, 2269 (2009)

[18] P.H. Yoon, R.C. Davidson, Phys. Rev. A 35, 2718 (1987)

[19] A. Bret, L. Gremillet, D. Benisti, E. Lefebvre, Phys. Rev. Lett. 100, 205008 (2008)

[20] H.H. Kaang, C.M. Ryu, P. Yoon, Phys. Plasmas 16, 082103 (2009)

[21] H.J. de Blank Phys. Plasmas 8, 3927 (2001)

[22] T.V.Liseikina, F. Pegoraro, E. Yu. Echkina, Phys. Plasmas 11, 3535 (2004)

[23] A. Ghizzo, T.W. Johnston, T. Reveille, P. Bertrand, M. Albrecht-Marc, Phys. Rev. E 74, 046407 (2006).

[24] A. Ghizzo, D. Del Sarto, T. Reveille, N. Besse, R. Klein, Phys. Plasmas 14, 062702 (2007).

[25] F. Califano, R. Prandi, F. Pegoraro, S.V. Bulanov, J. Plasma Physics 60, 331 (1998).

[26] A. Ghizzo, F. Huot, P. Bertrand, J. Comput. Phys. 186, 47 (2003). 